\title{
Conformational Studies of Proteoglycans: Theoretical Studies on the Conformation of Heparin
}

\author{
M. NAGARAJAN and V. S. R. RAO, Molecular Biophysics Unit, \\ Indian Institute of Science, Bangalore 560012, India
}

\begin{abstract}
Synopsis
Various models proposed for heparin have been examined by a stereochemical approach involving contact distance criteria and potential energy calculations. The present study suggests that the model favored by Atkins and coworkers [Biochem. J. (1973) 135, 729-733 and (1974) $143,251-252$ ] is not stereochemically satisfactory. An alternative model has been proposed with $N$-acetyl-D-glucosamine and one of the uronides in the ${ }^{4} \mathrm{C}_{1}$ conformation and the other uronide (probably sulfated) in the ${ }^{1} \mathrm{C}_{4}$ conformation. The observed variations in the tetrasaccharide periodicities (16.5-17.3 $\AA$ ) in different crystalline modifications of heparin have been attributed to possible changes in the rotational angles about the interunit glycosidic bonds rather than a change in the pyranose ring conformation. The proposed model is also independent of the observed variation in the relative composition of uronic acid residues in heparin. These conclusions are in disagreement with those of earlier workers.
\end{abstract}

Heparin is a highly sulfated, acidic heteropolysaccharide occurring in mammalian connective tissue. Under normal physiological conditions it occurs only intracellularly, in most cells or basophilic leukocytes. ${ }^{1}$ Pharmacologically it is important because of its anticoagulant properties and its role as a fat clearing agent. ${ }^{2-6}$

Until recently, heparin was thought to be composed of tetrasaccharide, or possibly disaccharide, repeating sequences with equimolar quantities of D-glucosamine and D-glucuronic acid residues, ${ }^{7,8}$ joined together with $\alpha(1 \rightarrow 4)$ glycosidic linkages. ${ }^{9}$ Recent chemical and physical studies ${ }^{10-15}$ not only revealed the presence of $L$-iduronic acid residues, but also indicated that this sugar moiety constitutes about $70-80 \%$ of the total uronic acid (UA) content. Lindahl and coworkers ${ }^{16,17}$ established the occurrence of $\beta$-glucuronidic linkages. However, the relative proportions of uronic acid composition vary depending on the source.

The number of sulfate groups in heparin also varies between four and six per tetrasaccharide unit. On the average, it contains approximately three sulfate groups per disaccharide; one of these is located on the amino group of glucosamine, the second is at C-6 (or C-3) of glucosamine, and the third is on the $\mathrm{C}-2$ atom of the iduronic acid moiety. ${ }^{17,18}$

Atkins and coworkers ${ }^{19-23}$ from x-ray diffraction studies on well-oriented fibers of a sodium salt of heparin obtained from hog intestinal mucosa and rat skin and a calcium salt of heparin suggested that in the solid state, heparin exists in three distinct conformations of tetrasaccharide periodicity 
of $16.5-16.8,17.3$, and $16.8 \AA$. For the sodium salt of heparin, these authors originally reported a value of $15.9 \AA$ for the tetrasaccharide repeat, but subsequently the value has been revised to $16.5 \AA$ (personal communication). Three models were considered for the molecular shape of the sodium salt of heparin, ${ }^{19}$ which differs mainly in the shape of the ring assumed for the uronic acid moieties (Fig. 1). In model I both the uronic acids were assumed to be in ${ }^{4} \mathrm{C}_{1}$ conformation. In model II one uronic acid was assumed to be in the ${ }^{4} \mathrm{C}_{1}$ chair form, whereas the other was in the ${ }^{1} \mathrm{C}_{4}$ conformation. And in model III both the uronic acids were assumed to be in the ${ }^{1} \mathrm{C}_{4}$ chair conformation. From various other considerations, these authors favored model III. It has also been pointed out that experimental data are insufficient to exclude any of these three models. ${ }^{19}$ Recently these authors ${ }^{23}$ also considered the possibility of skew boat conformation for iduronic acid residue in proposing the model for heparin. However, the energy calculations on hexuronic acids (unpublished results) clearly indicate that the ${ }^{1} \mathrm{C}_{4}$ conformation assumed by Nieduszynsky and Atkins ${ }^{19}$ for $\beta$-D-glucuronic acid and the ${ }^{1} \mathrm{~S}_{3}$ conformation for iduronic acid residues ${ }^{23}$
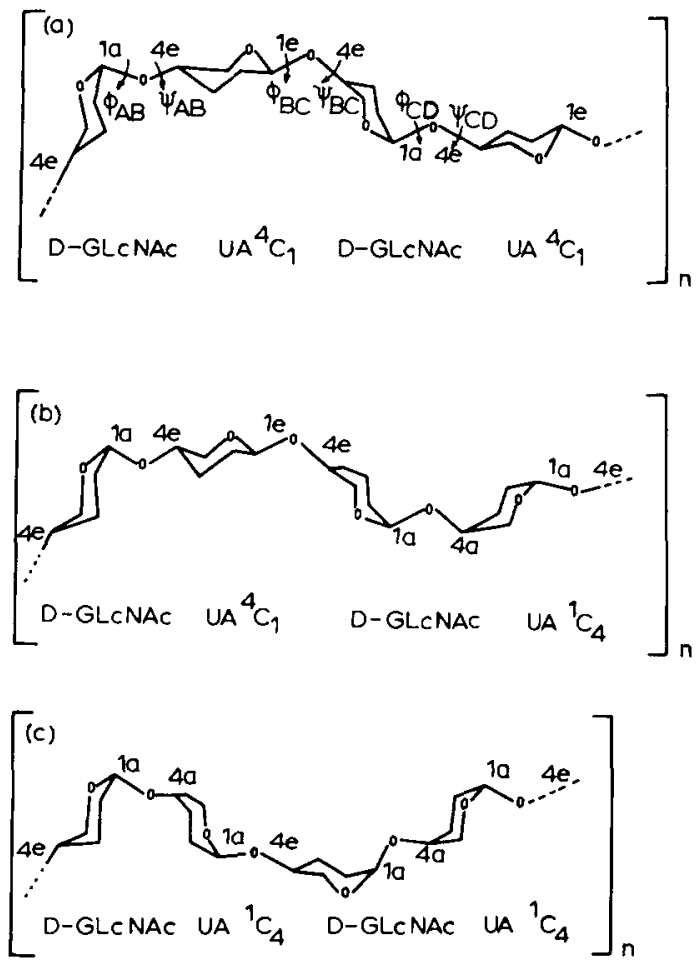

Fig. 1. Molecular conformations of heparin (Ref. 19). The repeating sugar residues are $\alpha$-N-acetyl-D-glucosamine and uronic acid, which may be either $\beta$-D-glucuronic acid or $\alpha$-L-iduronic acid joined through the $(1 \rightarrow 4)$ linkage. (a) A tetrasaccharide repeat with all sugar residues in the ${ }^{4} \mathrm{C}_{1}$ chair form. (b) One of the uronic acid residues in the ${ }^{1} \mathrm{C}_{4}$ chair form and others in the ${ }^{4} \mathrm{C}_{1}$ chair form. (c) Both uronic acid residues in the ${ }^{1} \mathrm{C}_{4}$ chair form. 
in the subsequent studies of heparin are not energetically favored (unpublished results). In fact the $x$-ray diffraction data ${ }^{23}$ could not discriminate the models built with ${ }^{1} \mathrm{C}_{4}$ and ${ }^{1} \mathrm{~S}_{3}$ conformers for iduronic acid residues. It thus seems that not only the composition of uronic acid and the location of sulfate groups, but also the conformation of the pyranose rings in the polymer are in doubt. Hence an attempt has been made to propose the probable models for the heparin obtained in different modifications from a stereochemical approach with the available information. Such a study throws light on the probable ring conformation of the uronide moieties, which is important for an understanding of its biological function.

\section{THEORETICAL CALCULATIONS}

\section{Fixing of Atoms}

Since $\mathrm{x}$-ray crystal structure analysis ${ }^{24,25}$ and theoretical studies ${ }^{26}$ have clearly established that $\alpha$-N-acetyl-D-glucosamine (GlcNAc) and $\beta$-D-glucuronic acid (GlcUA) favor the ${ }^{4} \mathrm{C}_{1}$ conformation, calculations were performed with this conformation for these monomer units. Since the energy difference between the ${ }^{4} \mathrm{C}_{1}$ and ${ }^{1} \mathrm{C}_{4}$ conformations of $\alpha$-L-iduronic acid (IdUA) is very small (unpublished results), both ring conformations ${ }^{4} \mathrm{C}_{1}$ and ${ }^{1} \mathrm{C}_{4}$ have been considered in the present study of this uronic acid moiety. The coordinates of $\beta$-glucuronic acid were obtained by using the standard values reported by Arnott and Scott. ${ }^{27}$ Since $\alpha$-L-iduronic acid and $\beta$-D-glucuronic acid, both in ${ }^{4} \mathrm{C}_{1}$ conformation, are C- 5 epimers, the coordinates of $\alpha$-L-iduronic acid were obtained by effecting the orientation of the substituent at the C-5 atom of the pyranose ring. The angle at the glycosidic bridge oxygen atom was assumed to be $117.5^{\circ}$. The acetamido group at the C-2 atom of $\mathrm{N}$-acetyl-D-glucosamine is assumed to be planar. The hydroxyl hydrogens, the atoms attached to the C-6 atom, and the sulfate groups are not fixed, since their positions are expected to vary with the environment. Similar assumptions were also made by Rees $^{28}$ in working out the allowed conformations of agar-carrageenin-chondroitin type of polymers, i.e., restriction of the polysaccharide conformation caused by ring atoms, and the $\mathrm{C}, \mathrm{O}, \mathrm{N}$, and $\mathrm{H}$ atoms bonded directly to those alone have been considered. The rotational angles $(\phi, \psi)$ specify the relative orientation of the adjacent residues, and their conventions are the same as those described earlier. ${ }^{29}$

\section{Energy Minimization of the Tetrasaccharide Fragment}

The total potential energy of the molecule was computed by considering nonbonded $V_{\text {nb }}$, electrostatic energy $V_{\text {es }}$, and the torsional energy terms $V_{\text {tor: }}$

where

$$
V_{\mathrm{tot}}=V_{\mathrm{nb}}+V_{\mathrm{es}}+V_{\mathrm{tor}}
$$




$$
\begin{gathered}
\text { NAGARAJAN AND RAO } \\
V_{\mathrm{nb}}=\epsilon_{i j}\left[F\left(\frac{\left\langle r_{g}\right\rangle_{i j}}{r_{i j}}\right)^{12}-2.0\left(\frac{\left\langle r_{g}\right\rangle_{i j}}{r_{i j}}\right)^{6}\right] \\
V_{\mathrm{es}}=332 \frac{q_{i} q_{j}}{\epsilon^{\prime} r_{i j}} \\
V_{\text {tor }}\left(\phi_{i}, \psi_{i}\right)=\frac{V_{\mathrm{rot}}}{2}\left(1+\cos 3 \phi_{i}\right)+\frac{V_{\mathrm{rot}}}{2}\left(1+\cos 3 \psi_{i}\right)
\end{gathered}
$$

In order to compute the electrostatic energy, the fractional charges on atoms of uronic acid and $\mathrm{N}$-acetyl-hexosamine were computed by treating the molecular fragment as comprising a $\sigma$ system and a separate $\pi$ system of bonds over the $\sigma$ frame. The $\pi$ charges due to the mobile electron density at each atom in the conjugated parts and the $\sigma$ charges on the saturated single bond framework were computed separately. To evaluate the $\sigma$ and $\pi$ charges, the MO-LCAO method of Del $\mathrm{Re}^{30}$ and the Huckel LCAO-MO methods ${ }^{31}$ were followed. To obtain the net charge on each atom, the $\sigma$ and $\pi$ charges were added. The charges so obtained were used for the calculation of electrostatic energy. The other parameters used in the above expressions are the same as those suggested by Scheraga and coworkers. ${ }^{32}$

The total potential energy of the tetrasaccharide is a function of six rotational angles $\left(\phi_{\mathrm{AB}}, \psi_{\mathrm{AB}}\right),\left(\phi_{\mathrm{BC}}, \psi_{\mathrm{BC}}\right)$, and $\left(\phi_{\mathrm{CD}}, \psi_{\mathrm{CD}}\right)$ (Fig. 1). It would be very complicated and time-consuming to search for the global minimum by considering all possible combinations of the three sets of dihedral angles. Hence the Fletcher-Powell-Davidson minimization method ${ }^{33,34}$ was followed to arrive at the global minimum. In this procedure a number of probable conformations were chosen with different sets of $\left(\phi_{\mathrm{AB}}, \psi_{\mathrm{AB}}\right),\left(\phi_{\mathrm{BC}}\right.$, $\left.\psi_{\mathrm{BC}}\right)$, and $\left(\phi_{\mathrm{CD}}, \psi_{\mathrm{CD}}\right)$, and all six variables were simultaneously allowed to vary to reach the local minimum on the six-dimensional energy surface. Among the local minima the lowest one was considered as the global minimum.

\section{RESULTS AND DISCUSSION}

The extreme-limit contour maps (which define the boundary of the allowed area) obtained using contact distance criteria ${ }^{35}$ for different disaccharide fragments in heparin are shown in Figs. 2-5:

1. $\alpha$-D-GlcNAc in the ${ }^{4} \mathrm{C}_{1}$ conformation joined through the $(1 \mathrm{a} \rightarrow 4 \mathrm{e})$ linkage to $\beta$-D-GlcUA in the ${ }^{4} \mathrm{C}_{1}$ conformation (Fig. 2).

2. $\beta$-D-GlcUA in the ${ }^{4} \mathrm{C}_{1}$ conformation joined through the $(1 \mathrm{e} \rightarrow 4 \mathrm{e})$ linkage to $\alpha$-D-GlcNAc in the ${ }^{4} \mathrm{C}_{1}$ conformation (Fig. 3).

3. $\alpha$-D-GlcNAc in ${ }^{4} \mathrm{C}_{1}$ conformation joined through the $(1 \mathrm{a} \rightarrow 4 \mathrm{e})$ linkage to $\alpha$-L-IdUA in the ${ }^{4} \mathrm{C}_{1}$ conformation (Fig. 2).

4. $\alpha$-L-IdUA in the ${ }^{4} \mathrm{C}_{1}$ conformation joined through the $(1 \mathrm{e} \rightarrow 4 \mathrm{e})$ linkage to $\alpha$-D-GlcNAc in the ${ }^{4} \mathrm{C}_{1}$ conformation (Fig. 3 ).

5. $\alpha$-D-GlcNAc in the ${ }^{4} \mathrm{C}_{1}$ conformation joined through the $(1 \mathrm{a} \rightarrow 4 \mathrm{a})$ linkage to $\alpha$-L-IdUA in the ${ }^{1} C_{4}$ conformation (Fig. 4). 


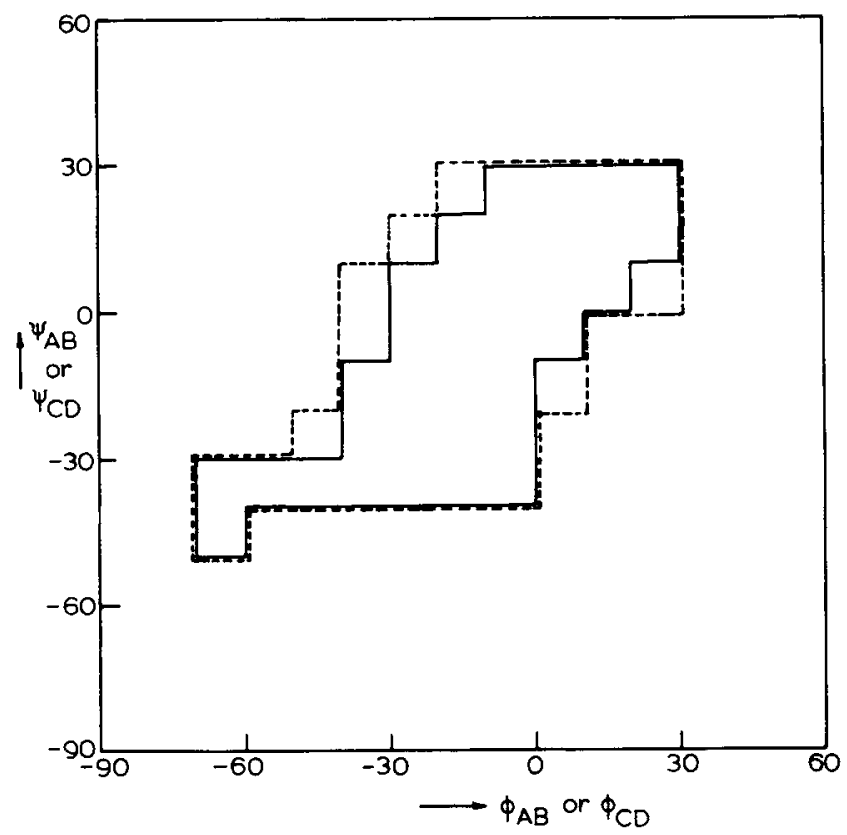

Fig. 2. Extreme-limit contour (-) for the disaccharide $\alpha$-D-GlcNAc joined through the $\left(1 \mathrm{a} \rightarrow 4 \mathrm{e}\right.$ ) linkage to $\beta$-D-GlcUA. Both residues are in the ${ }^{4} \mathrm{C}_{1}$ conformation (for $\tau=117.5^{\circ}$ ). Extreme-limit contour ( - - ) for the disaccharide $\alpha$-D-GIcNAc joined through the $(1 \mathrm{a} \rightarrow 4 \mathrm{e})$ linkage to the $\alpha$-L-IdUA. Both residues are in the ${ }^{4} \mathrm{C}_{1}$ conformation (for $\tau=117.5^{\circ}$ ).

6. $\alpha$-L-IdUA in the ${ }^{1} \mathrm{C}_{4}$ conformation joined through the $(1 \mathrm{a} \rightarrow 4 \mathrm{e})$ linkage to $\alpha$-D-GlcNAc in the ${ }^{4} \mathrm{C}_{1}$ conformation (Fig. 5).

Figure 3 shows that not only the allowed area, but also the shape of the extreme limit contours are nearly similar for the disaccharides $\beta$-D-GlcUA and $\alpha$-L-IdUA in the ${ }^{4} \mathrm{C}_{1}$ conformation joined through the $(1 \mathrm{e} \rightarrow 4 \mathrm{e})$ linkage to the GlcNAc residue, suggesting that the orientation of the $-\mathrm{COOH}$ group at the C-5 atom does not affect the allowed conformations significantly. The presence of a definite allowed area on the $(\phi, \psi)$ map indicates that these sugar residues in the ${ }^{4} \mathrm{C}_{1}$ conformation can be joined through the $(1 \rightarrow 4)$ linkage without any steric strain. However, the freedom of rotation of these residues is also highly restricted, as the allowed area constitutes only about $6 \%$ of the total area on the $\phi-\psi$ plane.

Since in proposing a model for heparin Nieduszynski and Atkins ${ }^{19}$ considered a twofold helix with a disaccharide repeat, two sets of rotational angles-i.e., one set of angles $\left(\phi_{\mathrm{AB}}, \psi_{\mathrm{AB}}\right)$ to define the conformation of the repeating disaccharide unit of GlcNAc-UA and $\left(\phi_{\mathrm{BC}}, \psi_{\mathrm{BC}}\right)$ to define the orientation of the adjacent repeating disaccharide units - are sufficient to represent such a helix. In such cases $\left(\phi_{\mathrm{AB}}, \psi_{\mathrm{AB}}\right)$ is same as $\left(\phi_{\mathrm{CD}}, \psi_{\mathrm{CD}}\right)$ (Fig. 1). Even such an approximation involves complications in the conformational analysis. In order to circumvent the complexity of the problem, one of the rotational angles $\left(\phi_{\mathrm{AB}}, \psi_{\mathrm{AB}}\right)$ has been fixed at one of its al- 


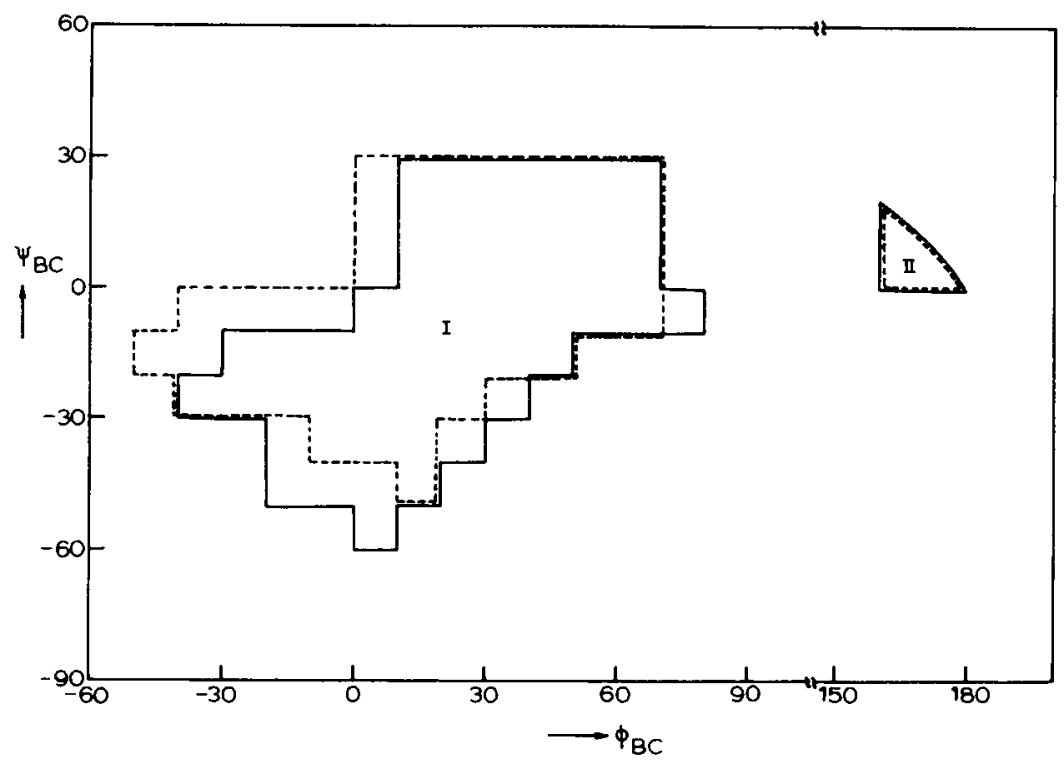

Fig. 3. Extreme-limit contour ( - ) for the disaccharide $\beta$-D-GlcUA joined through the (1e $\rightarrow 4 \mathrm{e}$ ) linkage to $\alpha$-D-GlcNAc. Both residues are in the ${ }^{4} \mathrm{C}_{1}$ conformation (for $\tau=117.5^{\circ}$ ). Extreme-limit contour (- -) for $\alpha$-L-IdUA joined through the (1e $\rightarrow 4 \mathrm{e})$ linkage to $\alpha$-D-GlcNAc. Both residues are in the ${ }^{4} \mathrm{C}_{1}$ conformation (for $\tau=117.5^{\circ}$ ).

lowed conformations and the heparin chain conformation has been studied by varying the angles $\left(\phi_{\mathrm{BC}}, \psi_{\mathrm{BC}}\right)$ at $10^{\circ}$ intervals. Next $\left(\phi_{\mathrm{AB}}, \psi_{\mathrm{AB}}\right)$ was fixed in a different allowed conformation, and the calculations were repeated. In this way all the possible conformations of heparin were explored. A similar approach has been used by different groups of workers analyzing the conformations of heteropolysaccharides. ${ }^{36,37}$

\section{Feasibility of Different Models Proposed by Earlier Workers}

In order to explain the observed tetrasaccharide repeat of $16.5 \AA$ for heparin, Nieduszynski and Atkins ${ }^{19}$ considered both the uronides in the ${ }^{4} \mathrm{C}_{1}$ and ${ }^{1} \mathrm{C}_{4}$ conformations and GIcNAc in the ${ }^{4} \mathrm{C}_{1}$ conformation and an approximate twofold helix with a covalent repeat approximating a disaccharide periodicity; the observed tetrasaccharide periodicity was assumed to result from the disposition of the sulfate groups (two on one glucosamine and one on the other). Hence this was considered as a helix with $n$ (number of disaccharide units per turn) $=2$ and $h$ (the projected height of the disaccharide about the helix axis) $=8.25 \AA$. Similarly, the tetrasaccharide repeats of 16.8 and $17.3 \AA$, respectively, correspond to helices with $n=2$ and $h=8.40 \AA$ and $n=2$ and $h=8.65 \AA$. 


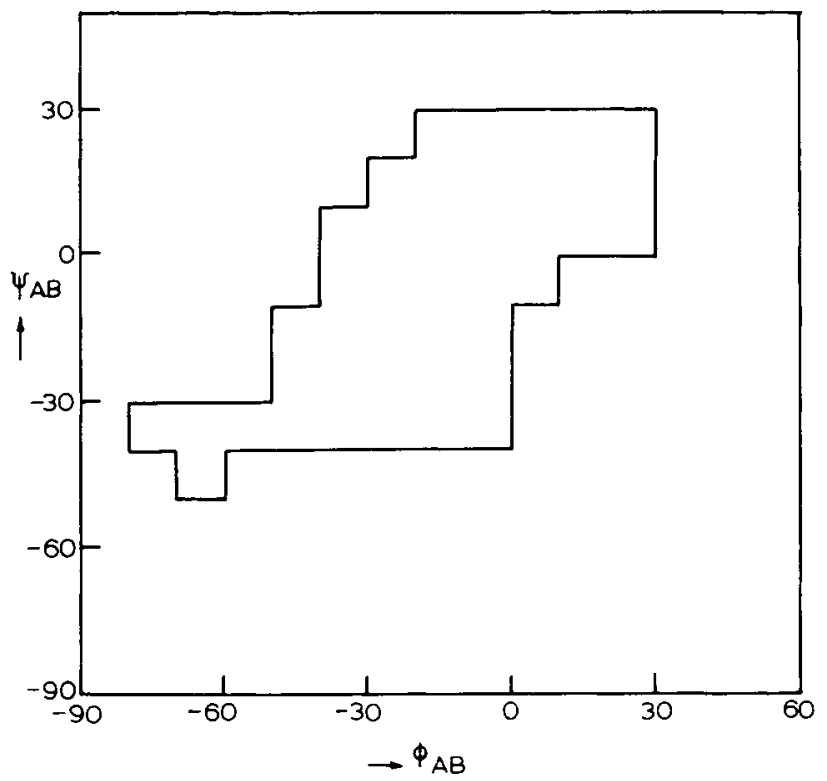

Fig. 4. Extreme-limit contour for $\alpha$-D-GlcNAc in the ${ }^{4} \mathrm{C}_{1}$ conformation joined through the $\left(1 \mathrm{a} \rightarrow 4 \mathrm{a}\right.$ ) linkage to $\alpha$-L-IdUA in the ${ }^{1} \mathrm{C}_{4}$ conformation (for $\tau=117.5^{\circ}$ ).

\section{Model I: Both Uronides in ${ }^{4} C_{1}$ Conformation}

In this case both uronides, i.e., $\beta$-D-GlcUA or $\alpha$-L-IdUA are assumed to be in the ${ }^{4} \mathrm{C}_{1}$ conformation. Figures 2 and 3 show small allowed regions indicating that disaccharide fragments: $\alpha$-D-GlcNAc in the ${ }^{4} \mathrm{C}_{1}$ conformation joined through the $(1 \mathrm{a} \rightarrow 4 \mathrm{e})$ linkage to $\alpha$-L-IdUA in the ${ }^{4} \mathrm{C}_{1}$ conformation and $\alpha$-L-IdUA in the ${ }^{4} \mathrm{C}_{1}$ conformation joined through $(1 \mathrm{e} \rightarrow 4 \mathrm{e})$ linkage to $\alpha$-D-GlcNAc in the ${ }^{4} \mathrm{C}_{1}$ conformation without steric strain. Since we are interested only in examining the models proposed by Atkins and coworkers, ${ }^{19-23}$ only those conformations which correspond to helical parameters $n=2$ and $h=8.25 \AA, n=2$ and $h=8.40 \AA$, and $n=2$ and $h=8.65$ $\AA$ have been examined.

By keeping $\left(\phi_{\mathrm{AB}}, \psi_{\mathrm{AB}}\right)$ in one of the allowed conformations, the conformational angles $\left(\phi_{\mathrm{BC}}, \psi_{\mathrm{BC}}\right)$ which satisfy the helical parameters $n=2$ and $h=8.25 \AA, n=2$ and $h=8.40 \AA$, and $n=2$ and $h=8.65 \AA$ were obtained by following the procedure described earlier; we found that none of these conformations are allowed. This suggests that the above type of helices ( $n=2$ and $h=8.25 \AA, n=2$ and $h=8.40 \AA$, and $n=2$ and $h=8.65 \AA$ ) considered by Atkins and coworkers ${ }^{19-23}$ do not satisfy the stereochemical criteria. This conclusion holds even if some of the $\alpha$-L-IdUA in the ${ }^{4} \mathrm{C}_{1}$ conformation are replaced by $\beta$-D-GlcUA in the ${ }^{4} \mathrm{C}_{1}$ conformation, since such changes do not affect the backbone conformations significantly. 


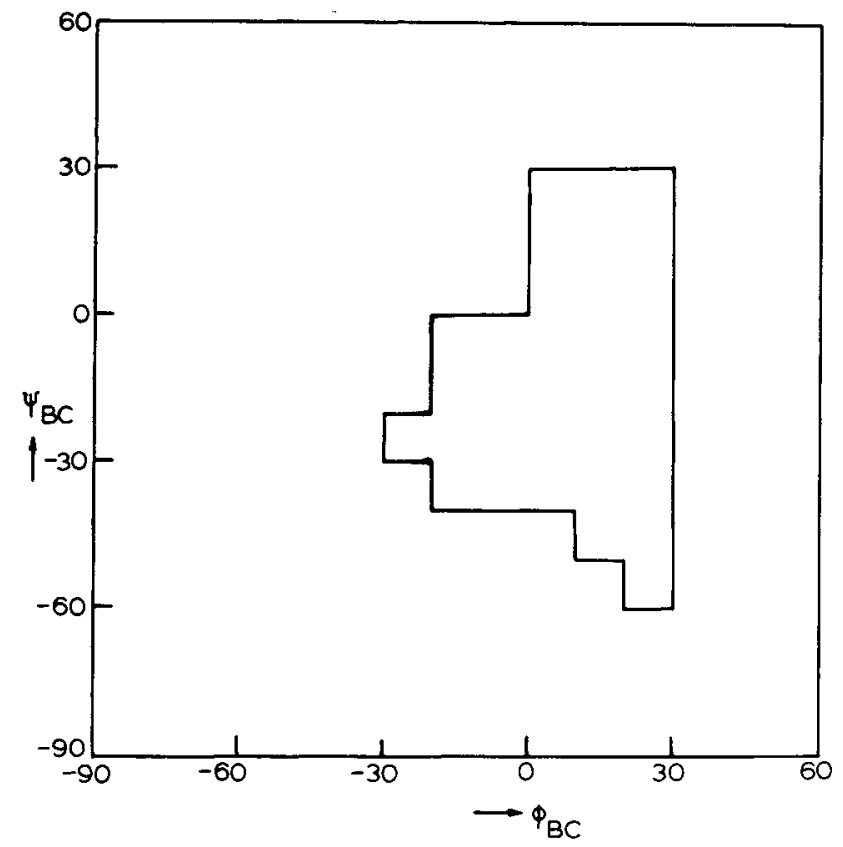

Fig. 5. Extreme-limit contour for $\alpha$-L-IdUA in the ${ }^{1} \mathrm{C}_{4}$ conformation joined through the $\left(\mathrm{la} \rightarrow 4 \mathrm{e}\right.$ ) linkage to $\alpha-\mathrm{D}-\mathrm{GlcNAc}$ in the ${ }^{4} \mathrm{C}_{1}$ conformation (for $\tau=117.5^{\circ}$ ).

\section{Model II. Uronic Acid Residue in ${ }^{1} \mathrm{C}_{4}$ Conformation}

As pointed out earlier, L-IdUA constitutes about $70-80 \%$ of the total uronic acid content. ${ }^{15}$ It remains unclear whether the two kinds of uronides, $\beta$-D-GlcUA and $\alpha$-L-IdUA, are randomly distributed or assembled in clusters. In the presence of certain salts, the blocks containing IdUA may perhaps crystallize preferentially. Since the energy difference between the ${ }^{4} \mathrm{C}_{1}$ and ${ }^{1} \mathrm{C}_{4}$ conformations is very small $\left(0.02 \mathrm{kcal} \mathrm{mol}{ }^{-1}\right)^{24}$ for $\alpha-\mathrm{L}$ IdUA, both ring conformations may coexist in solution. However, in the solid state, depending on the lattice energy, one may be preferred over the other. An $\mathrm{nmr}$ study ${ }^{14}$ also favors a ${ }^{1} \mathrm{C}_{4}$ conformation for $\alpha$-L-IdUA; hence a ${ }^{1} \mathrm{C}_{4}$ conformation has been assumed for $\alpha$-L-IdUA residues.

Figures 4 and 5 show the extreme-limit contour maps obtained for the following disaccharide fragments: $\alpha$-D-GlcNAc in the ${ }^{4} \mathrm{C}_{1}$ conformation joined through the $(1 \mathrm{a} \rightarrow 4 \mathrm{a})$ linkage to $\alpha$-L-IdUA in the ${ }^{1} \mathrm{C}_{4}$ conformation and $\alpha$-L-IdUA in the ${ }^{1} \mathrm{C}_{4}$ conformation joined through the $(1 \mathrm{a} \rightarrow 4 \mathrm{e})$ linkage to $\alpha$-D-GlcNAc in the ${ }^{4} \mathrm{C}_{1}$ conformation.

The allowed regions in these figures show that the sugar residues in these disaccharide fragments can be joined without steric strain. Since we are interested mainly in examining the models proposed by Atkins and coworkers, ${ }^{19-23}$ only those conformations which correspond to helical parameters $n=2$ and $h=8.25 \AA ; n=2$ and $h=8.40 \AA$; and $n=2$ and $h=8.65$ $\AA$ have been considered.

As mentioned earlier, by keeping $\left(\phi_{\mathrm{AB}}, \psi_{\mathrm{AB}}\right)$ in one of the allowed con- 
formations, the conformational angles $\left(\phi_{\mathrm{BC}}, \psi_{\mathrm{BC}}\right)$ which satisfy the helical parameters $n=2$ and $h=8.25 \AA$ are computed, and we found that none of the conformational angles $\left(\phi_{\mathrm{BC}}, \psi_{\mathrm{BC}}\right)$ so determined satisfy stereochemical criteria. The maximum theoretical extension for the disaccharide fragment $\alpha$-D-GlcNAc in the ${ }^{4} \mathrm{C}_{1}$ conformation joined through the (1a $\rightarrow$ 4a) linkage to $\alpha$-L-IdUA in the ${ }^{1} \mathrm{C}_{4}$ conformation is about $8.9 \AA$, and in reality this distance contracts to $7.7-8.3 \AA$ due to steric restrictions. Hence the twofold helices with axial repeat of 8.4 and $8.65 \AA$ cannot be explained by this present model.

These studies indicate that the twofold helical models with a disaccharide repeat considered by Atkins and coworkers ${ }^{19-23}$ can be ruled out by contact criteria alone.

\section{Models with a Tetrasaccharide Periodicity}

The sodium salt of heparin from hog intestinal mucosa crystallizes in a triclinic unit cell with a fiber (c) axis repeat of $16.5-16.8 \AA .19,23$ The axial repeats 16.5 and $16.8 \AA$ can be explained with four extended sugar residues. In other words, there is only one chain comprising a tetrasaccharide repeat passing through the unit cell. Since $x$-ray fiber data indicate that the gross features of the molecular shape are represented at the tetrasaccharide level, two models are considered to explain the observed tetrasaccharide repeat of $16.5-17.3 \AA$; they are as follows:

1. GlcNAc in the ${ }^{4} \mathrm{C}_{1}$ conformation and both uronides in the ${ }^{4} \mathrm{C}_{1}$ conformation.

2. GlcNAc in the ${ }^{4} \mathrm{C}_{1}$ conformation and one of the uronides in the ${ }^{4} \mathrm{C}_{1}$ conformation and the other uronide (probably sulfated) in the ${ }^{1} \mathrm{C}_{4}$ conformation.

The possibility of both uronic acid residues in the ${ }^{1} \mathrm{C}_{4}$ conformation has not been considered, since such a conformation would not satisfy the observed tetrasaccharide repeat.

\section{Model A}

In this model each sugar residue is in the ${ }^{4} \mathrm{C}_{1}$ conformation, and the glycosidic linkages are $(1 \mathrm{a} \rightarrow 4 \mathrm{e})$ between glucosamine and uronide and (1e $\rightarrow 4 \mathrm{e}$ ) between uronide and glucosamine [Fig. 1(a)].

Since Figs. 2 and 3 show that the allowed conformations are confined to one or two small regions, three sets of rotational angles were chosen for each $(\phi, \psi)$ as the starting sets for energy minimization: $\left(-50^{\circ},-40^{\circ}\right),\left(-10^{\circ}\right.$, $\left.-20^{\circ}\right)$, and $\left(20^{\circ}, 20^{\circ}\right)$ for $\left(\phi_{\mathrm{AB}}, \psi_{\mathrm{AB}}\right)$ (Fig. 2); $\left(50^{\circ}, 0^{\circ}\right),\left(10^{\circ},-30^{\circ}\right)$, and $\left(160^{\circ}, 10^{\circ}\right)$ for $\left(\phi_{\mathrm{BC}}, \psi_{\mathrm{BC}}\right)$ (Fig. 3); and $\left(-10^{\circ},-20^{\circ}\right),\left(-50^{\circ},-40^{\circ}\right)$, and $\left(20^{\circ}, 20^{\circ}\right)$ for $\left(\phi_{\mathrm{CD}}, \psi_{\mathrm{CD}}\right)$ (Fig. 2$)$.

The rotational angles of the initial and final conformations after minimization are shown in columns $2-4$ and 5-7 of Table I, respectively. The 


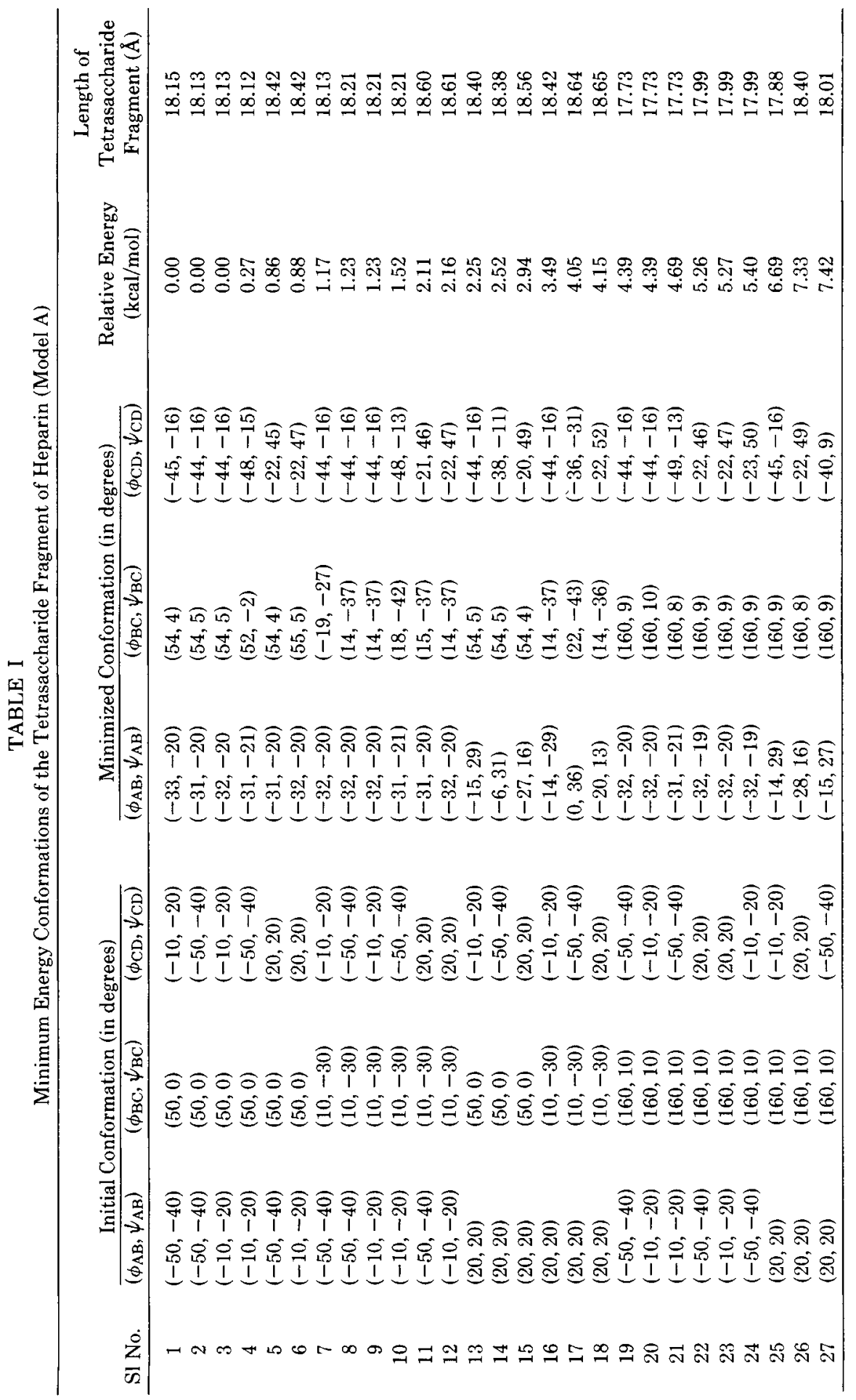


corresponding energy values and the lengths of the tetrasaccharide fragment are also shown in the last two columns of Table I. It is interesting to note that for the first few conformations (sets 1-18), where $\left(\phi_{\mathrm{BC}}, \psi_{\mathrm{BC}}\right)$ take up values in region I of the steric map (Fig. 3), the tetrasaccharide length varies between 18.0 and $18.6 \AA$ and the energy value ranges between 0 and $4 \mathrm{kcal} / \mathrm{mol}$. In contrast, when $\left(\phi_{\mathrm{BC}}, \psi_{\mathrm{BC}}\right)$ assume values in region II of the steric map (Fig. 3), the energy of the tetrasaccharide fragment increases and length of the fragment decreases to $17.7-18.0 \AA$. But the length of these tetrasaccharide fragments, 17.6-18.6 $\AA$, is much higher than the observed lengths of $16.5-17.3 \AA$.

\section{Model B}

This model differs from the previous model in that one of the two uronic acid residues, preferably a sulfated uronic acid residue, adopts the ${ }^{1} \mathrm{C}_{4}$ chair conformation [Fig. 1(b)]. All the three glycosidic linkages $(1 \mathrm{a} \rightarrow 4 \mathrm{e}),(1 \mathrm{e}$ $\rightarrow 4 \mathrm{e})$, and $(1 \mathrm{a} \rightarrow 4 \mathrm{a})$ are different in this model.

As in the previous case, a total of 27 conformations, 3 for each set of rotational angles (Figs. 2-4), were chosen as starting conformations for energy minimization. The rotational angles of the initial and minimized conformations are displayed in Table II. Those conformations whose minimized energy value is above $10 \mathrm{kcal} / \mathrm{mol}$ have not been shown in Table II. The energy of these conformations and the lengths of the tetrasaccharide fragment are also shown in Table II. The length of the tetrasaccharide fragment in all these cases varies from 16.3 to $17.3 \AA$, within the range of 16.5-17.3 $\AA$ observed for the sodium and calcium salts of heparin in the solid state.

It is interesting to note that in the first few conformations (sets 1-15), where $\left(\phi_{\mathrm{BC}}, \psi_{\mathrm{BC}}\right)$ assume values in the allowed region I of the steric map (Fig. 3), the tetrasaccharide length is about $16.9-17.3 \AA$ and the energy varies from $0-3.5 \mathrm{kcal} / \mathrm{mol}$. On the other hand, when $\left(\phi_{\mathrm{BC}}, \psi_{\mathrm{BC}}\right)$ assume values in region II of the extreme limit contour map (Fig. 3), the length of the tetrasaccharide decreases (16.3-16.9 $\AA$ ) and the energy value increases. Small changes in the angle at the bridge oxygen or geometry of the pyranose ring may slightly affect the length of the tetrasaccharide fragment. Hence the observed low periodicity of $16.5 \AA$ can also be explained by a small modification of the conformers (1-15), either by a small change in the geometry of the ring or the interunit glycosidic angle without assuming ( $\phi_{\mathrm{BA}}$, $\psi_{\mathrm{BC}}$ ) in region II of steric map (Fig. 3). It thus seems that the tetrasaccharide periodicities of 16.5-17.3 $\AA$ observed for different salts of heparin can be explained by this present model, i.e., by assuming the ${ }^{4} C_{1}$ conformation for GlcNAc and one of the uronides in the ${ }^{4} \mathrm{C}_{1}$ conformation and the other uronide (probably sulfated) in the ${ }^{1} \mathrm{C}_{4}$ conformation.

In conclusion, the present study indicates that the model proposed by Nieduszynski and Atkins ${ }^{19}$ is not satisfactory from stereochemical criteria. A model built up of GlcNAc and one of the uronides in the ${ }^{4} \mathrm{C}_{1}$ conformation 


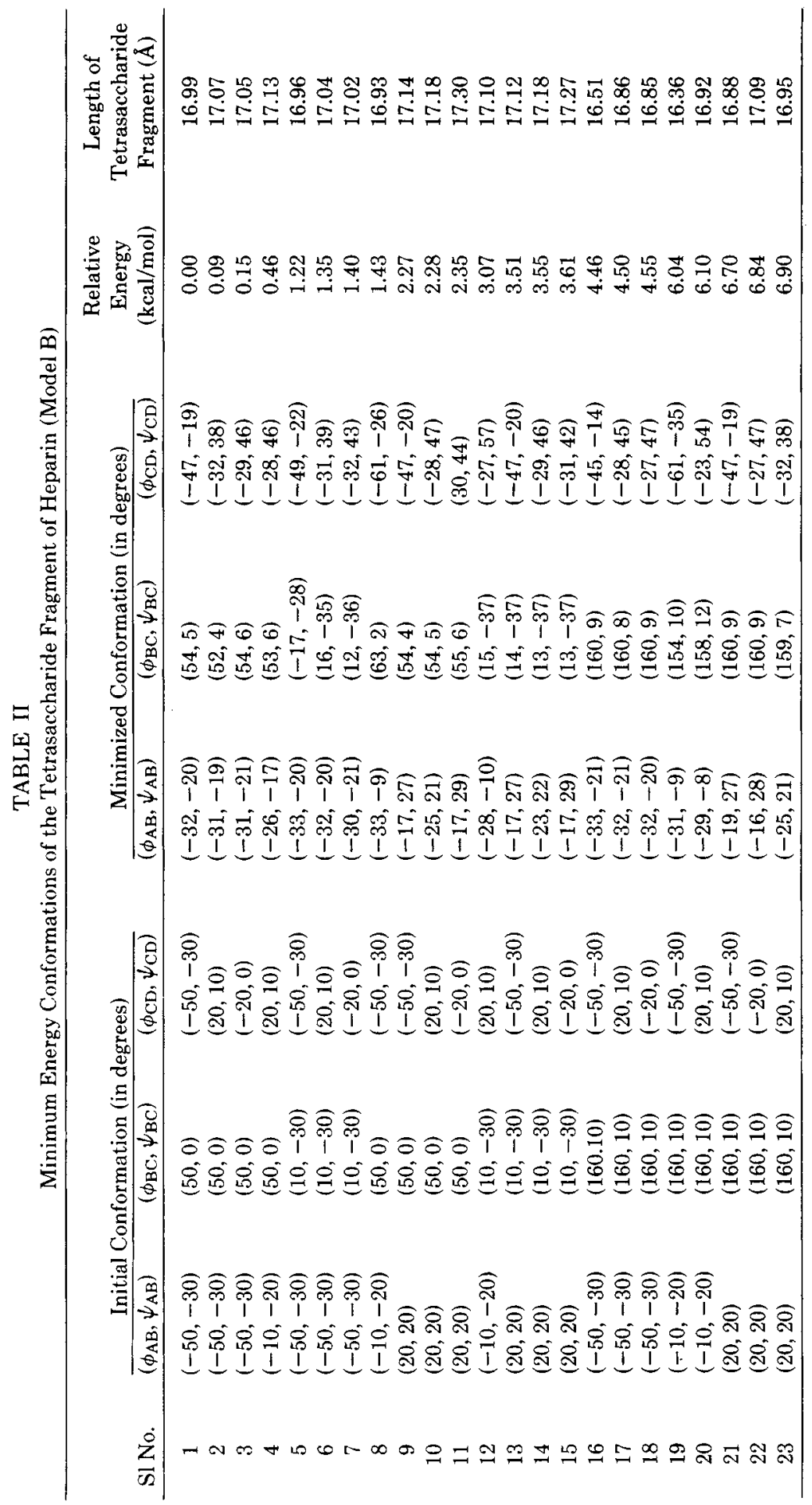


and the other in the ${ }^{1} \mathrm{C}_{4}$ conformation explains the observed repeats of 16.8-17.3 $\AA$. Replacement of $\beta$-D-glucuronic in the ${ }^{4} \mathrm{C}_{1}$ conformation by $\alpha$-L-induronic acid in the ${ }^{4} \mathrm{C}_{1}$ conformation affects only the orientation of the carboxyl group at C-5 but not the orientation of the interunit glycosidic bonds. As stated earlier, this change in the orientation of the - $\mathrm{COOH}$ group does not affect the allowed regions (Figs. 2 and 3) significantly. Hence the observed variation in the composition of UA affects only the orientation of the carboxyl group at C-5 atom but not the backbone conformation of heparin. This is in disagreement with the conclusions of earlier workers. ${ }^{19}$ Since the carboxyl group is also important for biological activity, these variations in the composition of UA may affect the biological activity.

One of us (M.N.) thanks the University Grants Commission, India for the award of a Junior Research Fellowship. This work was partially supported by the Department of Science and Technology, New Delhi.

Contribution No. 133 from the Molecular Biophysics Unit Indian Institute of Science, Bangalore, India.

\section{References}

1. Olsson, I., Berg, B., Fransson, L. A. \& Norden, A. (1970) Scand. J. Haematol. 7, $440-448$.

2. Bettleheim, F. A. (1970) in Biological Polyelectrolytes, Veis, A., Ed., Dekker, New York.

3. Janloz, R. W. (1970) in The Carbohydrates, Pigman, W. W. \& Horton, D., Eds., Academic, New York.

4. Lindahl, U. (1970) in Chemistry and Molecular Biology of Intercellular Matrix, Balasz, E. A., Ed., Academic, New York.

5. Ehrlich, J. \& Stivala, S. S. (1973) J. Pharm. Sci. 62, 517-544.

6. Engelberg, H. (1977) Fed. Proc. 36, 70-72.

7. Wolfrom, M. L., Weisblat, D. I., Karabinot, J. V., McNeely, W. H. \& McLean, J. (1943) J. Am. Chem. Soc. 65, 2077-2080.

8. Wolfrom, M. L., Tomomatsu, H. \& Szarek, W. A. (1966) J. Org. Chem., 31, 11731178.

9. Danishefsky, I. \& Steiner, H. (1965) Biochim. Biophys. Acta 101, 37-45.

10. Cifonelli, J. A. \& Dorfman, A. (1962) Biochem. Biophys. Res. Commun. 7 41-45.

11. Wolfrom, M. L., Wang, P. Y. \& Honda, S. (1969) Carbohydr. Res. 11, 179-185.

12. Lindahl, U. \& Axellson, O. (1971) J. Biol. Chem. 246, 74-82.

13. Perlin, A. S., Ng Ying Kin, N. M. K., Bhattacharjee, S. S. \& Johnson, L. F. (1972) Can.

J. Chem. 50, 2437-2441.

14. Perlin, A. S. (1977) Fed. Proc. 36, 106-109.

15. Hook, M., Lindahl, U. \& Iverius, P. H. (1974) Biochem. J. 137, 33-43.

16. Helting, T. \& Lindahl, U. (1971) J. Biol. Chem. 246, 5442-5447.

17. Lindahl, U., Backstrom, G., Malmstrom, A. \& Fransson, L. A. (1972) Biochem. Biophys. Res. Commun. 46, 985-991.

18. Elloway, H. F. \& Atkins, E. D. T. (1977) Biochem. J. 161, 495-498.

19. Nieduszynski, J. A. \& Atkins, E. D. T. (1973) Biochem. J. 135, 729-733.

20. Atkins, E. D. T., Nieduszynski, I. A. \& Horner, A. A. (1974) Biochem. J. 143, 251252.

21. Atkins, E. D. T. \& Nieduszynski, I. A. (1975) in Heparin-Structure, Function and Clinical Implication, Bradshaw, R. A. \& Wessler, S., Eds., Plenum, New York.

22. Atkins, E. D. T. \& Nieduszynski, I. A. (1977) Fed. Proc. 36, 78-83.

23. Nieduszynski, I. A., Gardner, K. H. \& Atkins, E. D. T. (1977) in Cellulose Chemistry and Technology, Arthur, J. C., Ed., American Chemical Society, Series 48. 
24. Johnson, L. N. (1966) Acta Crystallogr. 21, 885-891.

25. Gurr, G. H. (1964) Acta Crystallogr. 16, 690-696.

26. Virudachalam, R. \& Rao, V. S. R. (1976) Carbohydr. Res. 51, 135-139.

27. Arnott, S. \& Scott, W. E. (1972) J. Chem. Soc. Perkin Trans. II, 324-335.

28. Rees, D. A. (1969) J. Chem. Soc. B, 217-226.

29. Sathyanarayana, B. K. \& Rao, V. S. R. (1971) Biopolymers 10, 1605-1615.

30. Del Re, G., Pullman, B. \& Yonezawa, T. (1963) Biochim. Biophys. Acta 75, 153-182.

31. Pullman, B. \& Pullman, A. (1963) in Quantum Biochemistry, Interscience, New York.

32. Momany, F. A., Carruthers, L. M., McGuire, R. F. \& Scheraga, H. A. (1974) J. Phys. Chem. 78, 1595-1620.

33. Fletcher, R. \& Powell, M. J. D. (1963) Comput. J. 6, 163-168.

34. Davidson, W. C. (1959) AEC Research and Development Report, ANL-5990.

35. Ramachandran, G. N. \& Sasisekharan, V. (1968) Adv. Protein Chem. 23, 283-433.

36. Sathyanarayana, B. K. \& Rao, V. S. R. (1972) Biopolymers 11, 1379-1394.

37. Anderson, N. S., Campbell, J. W., Harding, M. M., Rees, D. A. \& Sanuel, J. W. B. (1969) J. Mol. Biol. 45, 85-99.

Received July 11, 1978

Returned for Revision September 18, 1978

Accepted December 1, 1978 